\title{
The Researches on Damage Detection Method for Truss Structures
}

\author{
Meng Hong WANG, Xiao Nan CAO \\ School of Civil and Transportation Engineering, Beijing University of Civil Engineering and Architecture, Beijing, China
}

\begin{abstract}
This paper presents an effective method to detect damage in truss structures. Numerical simulation and experimental analysis were carried out on a damaged truss structure under instantaneous excitation. The ideal excitation point and appropriate hammering method were determined to extract time domain signals under two working conditions. The frequency response function and principal component analysis were used for data processing, and the angle between the frequency response function vectors was selected as a damage index to ascertain the location of a damaged bar in the truss structure. In the numerical simulation, the time domain signal of all nodes was extracted to determine the location of the damaged bar. In the experimental analysis, the time domain signal of a portion of the nodes was extracted on the basis of an optimal sensor placement method based on the node strain energy coefficient. The results of the numerical simulation and experimental analysis showed that the damage detection method based on the frequency response function and principal component analysis could locate the damaged bar accurately.
\end{abstract}

\section{Introduction}

With the development of prefabrication and the mechanized construction of truss structures, more and more buildings are adopting a truss structure. However, the weight of equipment is increasing. Under the long-term action of moving loads, bolts loosen, rods bend and trusses fracture due to local damage, which can easily lead to a catastrophic accident. It is difficult to locate damage owing to the large number of structural members. Therefore, it is very important to identify the location of damage in a truss structure accurately.

Adams [1] and Cowley have detected damage in structures by collecting parameters related to frequency, accurately determining the damage location by using the ratio of the corresponding frequency changes before and after the structure was damaged. Numerical simulation of a simple cable has been carried out by Zheng Minggang et al. [2], frequency response function (FRF) curvature has been used and who found that after a damage index had been selected, FRF curvature could determine the damage location and damage degree sensitively. Yang and $\mathrm{Wu}[3]$ detected damage in a simple model using the acceleration frequency response function. The analysis of a combined T-shape beam was carried out by Zhang [4], who used the difference between the matrices formed by the frequency response function of the structure before and after damage as the basic parameter. At the same time, principal component analysis (PCA) was used for dimensionality reduction. Numerical analysis of the cantilever beam model was carried out by $\mathrm{Li}$ and $\mathrm{Yu}$ [5], who detected damage using the frequency response function of the structure and achieved satisfactory accuracy of damage identification.

There has been many research on structural damage detection based on the frequency response function, but most of the research is aimed at the simply-supported beam, cantilever beam and bridge structures. Research on damage detection in truss structures is scarce. Therefore, in this paper, a method of damage detection in truss structures will be researched by means of numerical simulation and experimental analysis.

\section{Basic Theory}

\subsection{Frequency Response Function}

The transfer function is the ratio between the output signal and the input signal in a structure and is one of the functions used most widely to describe the vibration characteristics of a structure.

After Laplace transformation, the multi-freedom vibration equation can be simplified as:

$$
Y(s)=H(s) F(s)
$$

In the equation, $Y(s)$ is the output signal; $F(s)$ is the input signal.

After Fourier transformation, the above equation can be written as follows:

$$
H(\omega)=\frac{Y(\omega)}{F(\omega)}
$$

* Corresponding author: wangmh@bucea.edu.cn 
Where $H(\omega)$ is the frequency response function.

\subsection{Principal Component Analysis(PCA)}

Assuming that there are $\mathrm{n}$ samples, and each sample contains m indexes, the original data matrix $X_{n \times m}$ can be obtained. The new matrix $Y_{n \times m}$ will be obtained after the original data matrix $X_{n \times m}$ is standardized. Subsequently, for the $\mathrm{n}$ samples given, the covariance matrix is obtained for the new matrix $Y_{n \times m}$; each element of the covariance matrix is represented by its corresponding covariance. The covariance matrix is a real symmetric matrix; therefore, it has $\mathrm{m}$ eigenvalues. Assuming that the eigenvalues are $\lambda_{1}, \lambda_{2}, \Lambda, \lambda_{m}$ $\left(\lambda_{1} \geq \lambda_{2} \geq \lambda_{3} \geq \Lambda \geq \lambda_{m}\right.$ ) the normalized feature vectors corresponding to the $\mathrm{m}$ eigenvalues are $u_{1}, u_{2}$, $\Lambda . u_{m}$. Finally, these feature vectors are used as the coefficient vectors of the principal components, then, combined with the standard matrix $Y_{n \times m}$, each principal component $Z_{1}, Z_{2} \wedge \wedge, Z_{m}$ of the original data can be obtained:

$$
\left\{\begin{array}{l}
Z_{1}=u_{11} Y_{1}+u_{12} Y_{2}+\Lambda+u_{1 m} Y_{m} \\
Z_{2}=u_{21} Y_{1}+u_{22} Y_{2}+\Lambda+u_{2 m} Y_{m} \\
\Lambda \Lambda \Lambda \Lambda \\
Z_{m}=u_{m 1} Y_{1}+u_{m 2} Y_{2}+\Lambda+u_{m m} Y_{m}
\end{array}\right.
$$

The cumulative contribution rate of the principal components $Z_{1}, Z_{2} \wedge \Lambda, Z_{m}$ should exceed $85 \%$ :

$$
\sum_{i=1}^{p} Z_{i} / \sum_{i=1}^{m} Z_{i} \geq 85 \%
$$

\section{Numerical Simulation}

\subsection{Numerical Simulation Model and Working Condition Setting}

Parameters of the model are as follows: The span of the truss structure is $3 \mathrm{~m} \times 5 \mathrm{~m}$, the truss structure contains 60 nodes and 174 rods and the size of all member sections is $\Phi 42 \mathrm{~mm} \times 2.5 \mathrm{~mm}$. Q235 steel was selected, the elastic modulus is $\mathrm{E}=200 \mathrm{GPa}$. In the numerical simulation, the self-weight of the rod was transferred into the equivalent node load without considering dust, snow or wind loads. Two conditions are defined in this paper. Condition I: no damage to any members; Condition II: damage has just occurred in rod 34; the ends of the rod are node 7 and node 16, as shown in Figure 1.

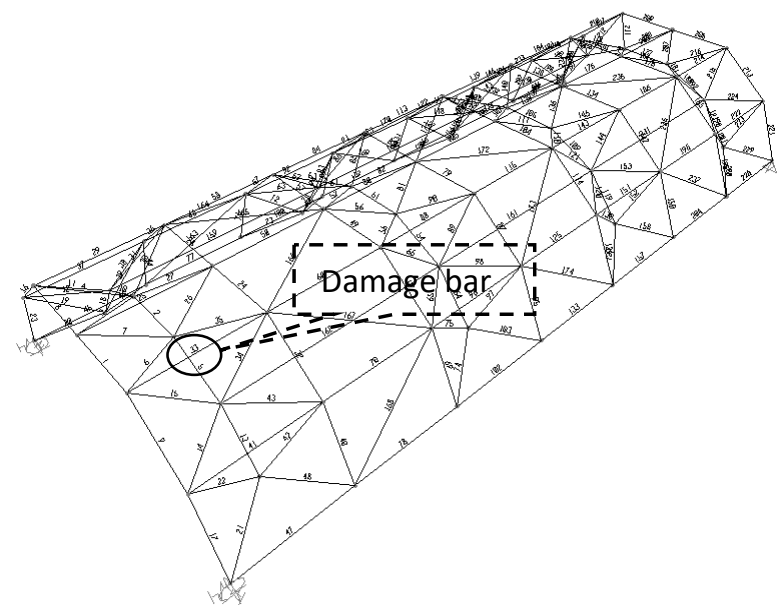

Figure 1 Damage Location

frequency domain signal of each node, and the frequency response function matrix was composed of all of the column vectors.

The frequency response function matrix was input to the statistical analysis software SPSS, in which principal component analysis (PCA) was applied to reduce the dimension of the matrix. The results of the dimensionality reduction under the two working conditions are shown in Tables 1 and 2, respectively.
In this paper, the finite element analysis software SAP2000 was used for numerical analysis. The sampling frequency was $512 \mathrm{~Hz}$ and the sampling time was $2 \mathrm{~s}$. Using MATLAB, frequency domain signals were derived from the time domain signals based on the Fourier transform. A column vector was made up of the

Table 1 Result of Dimensionality Reduction Under Condition I

Total Variance Explained 


\begin{tabular}{cccc|ccc}
\hline Component & Total & \% of Variance & Cumulative \% & Total & \% of Variance & Cumulative \% \\
\hline 1 & 717.269 & 69.977 & 69.977 & 717.269 & 69.977 & 69.977 \\
2 & 178.833 & 17.447 & 87.425 & 178.833 & 17.447 & 87.425 \\
3 & 74.087 & 7.228 & 94.653 & 74.087 & 7.228 & 94.653 \\
4 & 39.358 & 3.840 & 98.492 & 39.358 & 3.840 & 98.492 \\
5 & 8.969 & .875 & 99.367 & 8.969 & .875 & 99.367 \\
6 & 3.428 & .334 & 99.702 & 3.428 & .334 & 99.702 \\
\hline
\end{tabular}

Table 2 Result of Dimensionality Reduction Under Condition II

\begin{tabular}{|c|c|c|c|c|c|c|}
\hline \multicolumn{7}{|c|}{$\begin{array}{r}\text { Total Variance Explained } \\
\end{array}$} \\
\hline & \multicolumn{3}{|c|}{ Initial Eigenvalues } & \multicolumn{3}{|c|}{ Extraction Sums of Squared and Loading } \\
\hline Component & Total & $\%$ of Variance & Cumulative \% & Total & $\%$ of Variance & Cumulative $\%$ \\
\hline 1 & 680.221 & 66.363 & 66.363 & 680.221 & 66.363 & 66.363 \\
\hline 2 & 177.148 & 17.283 & 83.646 & 177.148 & 17.283 & 83.646 \\
\hline 3 & 60.005 & 5.854 & 89.500 & 60.005 & 5.854 & 89.500 \\
\hline 4 & 34.181 & 3.335 & 92.835 & 34.181 & 3.335 & 92.835 \\
\hline 5 & 25.535 & 2.491 & 95.326 & 25.535 & 2.491 & 95.326 \\
\hline 6 & 15.042 & 1.467 & 96.793 & 15.042 & 1.467 & 96.793 \\
\hline 7 & 9.811 & .957 & 97.750 & 9.811 & .957 & 97.750 \\
\hline 8 & 6.711 & .655 & 98.405 & 6.711 & .655 & 98.405 \\
\hline
\end{tabular}

From Table 1 and Table 2, we can see that the cumulative variance contribution rate of the third matrix component under the two working conditions has exceeded $85 \%$. Therefore, extraction of the first three principal components meets the requirement for dimensionality reduction. In SPSS, using the method of dimensionality reduction by principal component analysis, the first three principal component coefficients can be derived automatically, and then, the principal components $Z_{1}, Z_{2} \wedge, Z_{P}$ can be calculated.

\subsection{Damage Index}

According to the results of the numerical simulation, the frequency domain signals of each node can be regarded as a three dimensional vector before and after the damage. The angle between the two vectors before and after the damage was selected as the damage index. In the analysis of the results, if the angle between the two vectors before and after the damage of a node takes a maximum value, it can be concluded that the damage occurred in the vicinity of the node.

To make the simulation results clearer, the difference between the FRF vector angle and the upper confidence limit was used to judge damage location in this paper. If the calculated result was negative, then the difference was taken to be zero.

\subsection{Numerical Simulation Analysis}

The difference between the FRF vector angle and the upper confidence limit is shown in Figure 2. As can be seen in Figure 2, the difference between the FRF vector angle and the upper confidence limit took its maximum values at node 7 and node 16, namely, damage occurred in the vicinity of node 7 and node 16 . Therefore, it could be estimated that rod 34, which connected the two nodes, had been damaged.

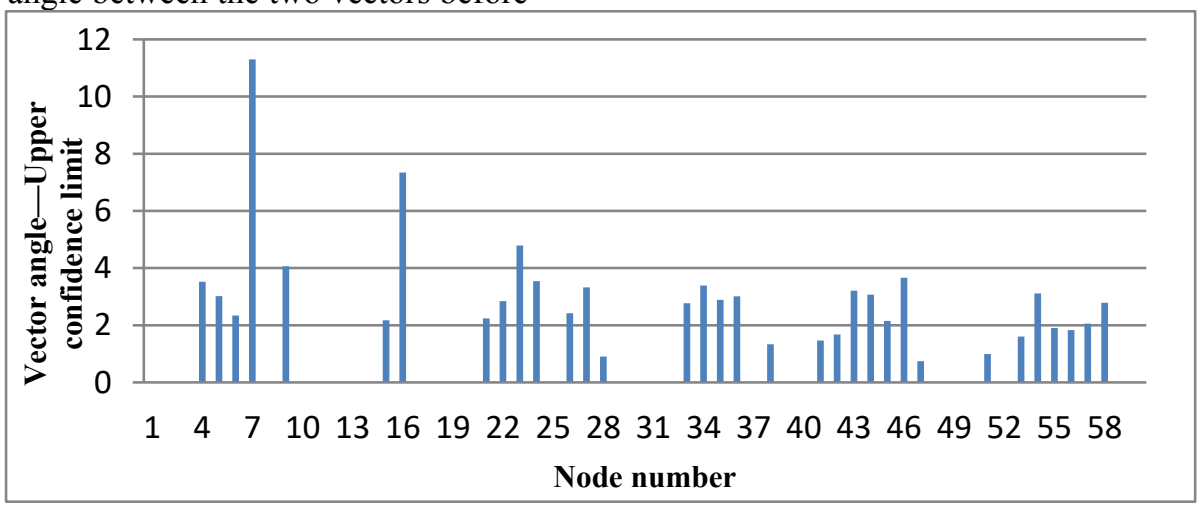

Figure 2 Damage Detection Result Under Condition II

\section{Experimental Analysis}

\subsection{Experimental Procedure}

In this paper, the working conditions and the size of the experimental model was the same as that of the finite element numerical simulation model. In this experiment, damage detection on the truss structure was divided into two steps: First, with the sensors arranged as shown in Figure 4, the time domain signal of 20 nodes was obtained by the hammering method. After data processing, we found the suspected damage nodes through the identification result obtained in the first step. Second, based on the suspected damage nodes from the first step, the scope of damage identification was narrowed down to the nodes linked with the suspected 
damage nodes, and the specific location of the damaged bars was determined. The test method of single-point excitation and multi-point output was used. Node 19, as shown in Figure 3, was selected as the excitation point.

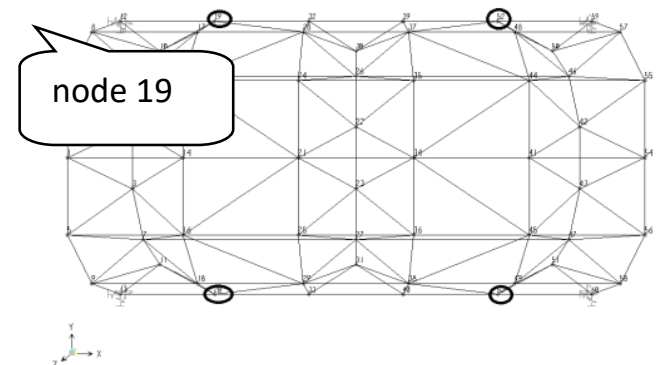

Figure 3 Excitation Node

\subsection{Experimental Results}

The first step: The FRF matrix was input into statistical analysis software SPSS and the dimensionality was reduced using PCA. The results of dimensionality reduction are shown in Table 3 and Table 4. From Table

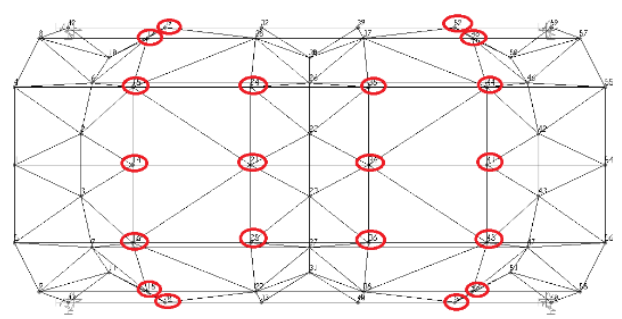

Figure 4 Sensor Arrangement

3 and Table 4, we can see that the cumulative variance contribution rate of the 11th matrix component under the two working conditions exceeded $85 \%$; therefore, the first 11 principal components of the FRF were extracted, then, according to equation (3), the principal components $Z_{1} 、 Z_{2} \wedge, Z_{P}$ were calculated.

Table 3 Result of Dimensionality Reduction Under Condition I

\begin{tabular}{|c|c|c|c|c|c|c|}
\hline \multicolumn{7}{|c|}{ Total Variance Explained } \\
\hline & \multicolumn{3}{|c|}{ Initial Eigenvalues } & \multicolumn{3}{|c|}{ Extraction Sums of Squared and Loading } \\
\hline Component & Total & $\%$ of Variance & Cumulative \% & Total & $\%$ of Variance & Cumulative $\%$ \\
\hline 1 & 100.670 & 19.662 & 19.662 & 100.670 & 19.662 & 19.662 \\
\hline 2 & 60.527 & 11.822 & 31.484 & 60.527 & 11.822 & 31.484 \\
\hline 3 & 49.786 & 9.724 & 41.208 & 49.786 & 9.724 & 41.208 \\
\hline 4 & 47.151 & 9.209 & 50.417 & 47.151 & 9.209 & 50.417 \\
\hline 5 & 38.138 & 7.449 & 57.866 & 38.138 & 7.449 & 57.866 \\
\hline 6 & 36.437 & 7.117 & 64.982 & 36.437 & 7.117 & 64.982 \\
\hline 7 & 26.678 & 5.211 & 70.193 & 26.678 & 5.211 & 70.193 \\
\hline 8 & 23.720 & 4.633 & 74.826 & 23.720 & 4.633 & 74.826 \\
\hline 9 & 19.772 & 3.862 & 78.687 & 19.772 & 3.862 & 78.687 \\
\hline 10 & 18.528 & 3.619 & 82.306 & 18.528 & 3.619 & 82.306 \\
\hline 11 & 15.861 & 3.098 & 85.404 & 15.861 & 3.098 & 85.404 \\
\hline 12 & 14.127 & 2.759 & 88.163 & 14.127 & 2.759 & 88.163 \\
\hline
\end{tabular}

Table 4 Result of Dimensionality Reduction Under Condition II

\begin{tabular}{|c|c|c|c|c|c|c|}
\hline \multicolumn{7}{|c|}{ Total Variance Explained } \\
\hline & \multicolumn{3}{|c|}{ Initial Eigenvalues } & \multicolumn{3}{|c|}{ Extraction Sums of Squared and Loading } \\
\hline Component & Total & $\%$ of Variance & Cumulative $\%$ & Total & $\%$ of Variance & Cumulative $\%$ \\
\hline 1 & 88.848 & 17.353 & 17.353 & 88.848 & 17.353 & 17.353 \\
\hline 2 & 59.473 & 11.616 & 28.969 & 59.473 & 11.616 & 28.969 \\
\hline 3 & 52.610 & 10.275 & 39.244 & 52.610 & 10.275 & 39.244 \\
\hline 4 & 50.236 & 9.812 & 49.056 & 50.236 & 9.812 & 49.056 \\
\hline 5 & 38.182 & 7.457 & 56.514 & 38.182 & 7.457 & 56.514 \\
\hline 6 & 32.147 & 6.279 & 62.792 & 32.147 & 6.279 & 62.792 \\
\hline 7 & 30.520 & 5.961 & 68.753 & 30.520 & 5.961 & 68.753 \\
\hline 8 & 27.994 & 5.468 & 74.221 & 27.994 & 5.468 & 74.221 \\
\hline 9 & 25.622 & 5.004 & 79.225 & 25.622 & 5.004 & 79.225 \\
\hline 10 & 18.122 & 3.540 & 82.765 & 18.122 & 3.540 & 82.765 \\
\hline 11 & 17.141 & 3.348 & 86.113 & 17.141 & 3.348 & 86.113 \\
\hline 12 & 13.606 & 2.657 & 88.770 & 13.606 & 2.657 & 88.770 \\
\hline
\end{tabular}

The difference between the FRF vector angle and the upper confidence limit based on the principal components calculated in the first step is shown in Figure 5. It can be seen from Figure 5 that the difference reached its maximum at the node 16 ; therefore, it was concluded that damage had occurred in the vicinity of node 16. 


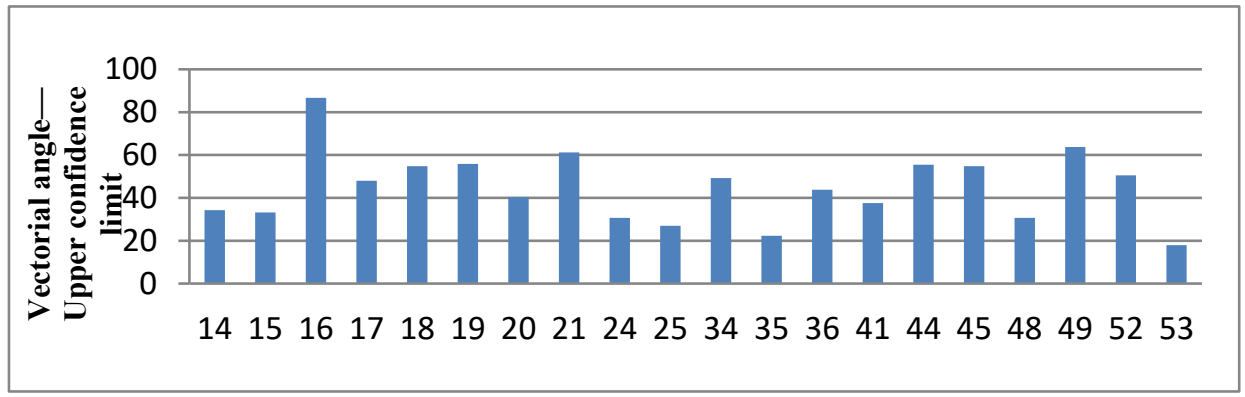

Figure 5 Identification Result of the First Step

The second step: The nodes connected to node 16 are numbered 3, 7, 11, 14, 18, 21 and 29. Time domain signals of the 7 nodes before and after the damage were acquired, the FRF matrix was input into statistical analysis software SPSS and the dimensionality was reduced using PCA. The results of dimensionality reduction are shown in Table 5 and Table 6 . From Table 5 and Table 6 , we can see that the cumulative variance contribution rate of the 5th matrix component has exceeded $85 \%$; therefore, the first five principal components of the FRF were extracted, then, the principal components $Z_{1}, Z_{2}{ } \wedge, Z_{P}$ were calculated.

Table 5 Result of Dimensionality Reduction Under Condition I

\begin{tabular}{|c|c|c|c|c|c|c|}
\hline \multicolumn{7}{|c|}{ Total Variance Explained } \\
\hline & \multicolumn{3}{|c|}{ Initial Eigenvalues } & \multicolumn{3}{|c|}{ Extraction Sums of Squared and Loading } \\
\hline Component & Total & $\%$ of Variance & Cumulative $\%$ & Total & $\%$ of Variance & Cumulative $\%$ \\
\hline 1 & 242.035 & 47.272 & 47.272 & 242.035 & 47.272 & 47.272 \\
\hline 2 & 96.026 & 18.755 & 66.028 & 96.026 & 18.755 & 66.028 \\
\hline 3 & 60.220 & 11.762 & 77.789 & 60.220 & 11.762 & 77.789 \\
\hline 4 & 52.513 & 10.256 & 88.046 & 52.513 & 10.256 & 88.046 \\
\hline 5 & 38.374 & 7.495 & 95.541 & 38.374 & 7.495 & 95.541 \\
\hline
\end{tabular}

Table 6 Result of Dimensionality Reduction Under Condition II

\begin{tabular}{|c|c|c|c|c|c|c|}
\hline \multicolumn{7}{|c|}{ Total Variance Explained } \\
\hline & \multicolumn{3}{|c|}{ Initial Eigenvalues } & \multicolumn{3}{|c|}{ Extraction Sums of Squared and Loading } \\
\hline Component & Total & $\%$ of Variance & Cumulative $\%$ & Total & $\%$ of Variance & Cumulative $\%$ \\
\hline 1 & 148.089 & 28.924 & 28.924 & 148.089 & 28.924 & 28.924 \\
\hline 2 & 103.497 & 20.214 & 49.138 & 103.497 & 20.214 & 49.138 \\
\hline 3 & 86.298 & 16.855 & 65.993 & 86.298 & 16.855 & 65.993 \\
\hline 4 & 65.859 & 12.863 & 78.856 & 65.859 & 12.863 & 78.856 \\
\hline 5 & 63.479 & 12.398 & 91.254 & 63.479 & 12.398 & 91.254 \\
\hline
\end{tabular}

The difference between the FRF vector angle and the confidence upper limit based on the calculated principal

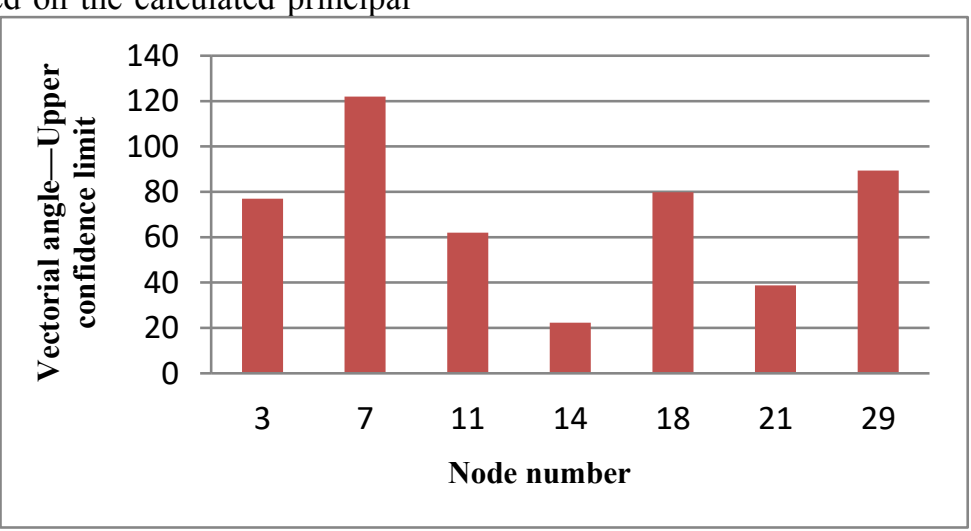

Figure 6 Identification Result of the Second Step

It can be seen from Figure 6 that the difference reached its maximum at node 7 ; therefore, damage was judged to have occurred in the vicinity of node 7 . Combining this result with the identification result of the first step, damage occurred in the vicinity of nodes 7 and 16 and in bar 34, which was linked with nodes 7 and 16 . The damage position was consistent with the experimental setup, and the result of the two step method was accurate.

\section{Conclusion}

This paper studied a damage detection method for a truss structure based on principal component analysis and the frequency response function. The accuracy of this 
method was verified by comparing the results of numerical simulation and experimental analysis. Conclusions can be drawn as follows:

(1) The structure was excited using the hammering method, and the time domain signal of the structure was extracted. The results showed that the measurement error can be reduced effectively by calculating the average value.

(2) Principal component analysis and the frequency response function were used to process all data, and numerical simulation and experimental analysis verified the effectiveness of the damage detection method. The method and its theoretical basis were provided for the study of similar problems.

(3) In the numerical simulation, time domain signals were extracted for all nodes and frequency domain signals were derived from the time domain signals based on the Fourier transform. After dimensionality reduction by SPSS, the angle between the two vectors before and after the damage was selected as the damage index. The damage location on the truss structure could be determined by the damage index in one step. The accuracy of the damage detection method has been verified.

(4) In experimental analysis, time domain signals of 20 nodes were collected using the method of optimal sensor placement based on the node strain energy participation coefficient, and then damage location detection was divided into two steps in which the scope of the damage range was narrowed gradually. The sensitivity and accuracy of the damage detection method was verified by the experimental results. Furthermore, the method has the prospect of application in practical engineering.

\section{Acknowledgement}

The authors would like to acknowledge the financial support provided by the National Natural Science Foundation of China (51578038) and Beijing National Science Foundation (8132023).

\section{References}

1. Cowley P, Adams RD. The location of defects in structures from measurements of natural frequencies. Journal of Strain Analysis 1979; 14(2): 49-57.

2. Zheng MG, Liu TX, Chen ZN. Structural damage assessment based on frequency response function. Mechanical Science and Technology 2001; 20(3): 479-481.

3. Yang HF, Wu ZY, Wu D. Structural damage detection method based on acceleration frequency response function. Journal of Vibration and Shock 2007; 26(2). 90-97.

4. Zhang $\mathrm{CH}$. Study on damage identification of structures based on principal component analysis. Chongqing: Chongqing Jiaotong University;2012.

5. Li XP, Yu ZW. Structural damage identification based on frequency response function. Journal of
China \& Foreign Highway 2006; 26(1). 83-85.

6. Chen Y. Research on sensor optimal placement and programmatic implementation of health monitoring on truss structures. Beijing: Beijing University of Civil Engineering and Architecture; 2014.

7. Sun HM, Li HN. State-of-the-art review of the structural health monitoring in civil engineering. Journal of Disaster Prevention and Mitigation Engineering 2003; 23(3): 92-98.

8. Beijing Jintuwu Software Technology Co., Ltd. Guidance to use of SAP2000 in Chinese. Beijing, China: China Communications Press; 2012.

9. Yan D, Qi CX, Feng QH. SAP2000 structure engineering analysis and example explanation. Beijing, China: China Architecture and Building Press; 2009. 